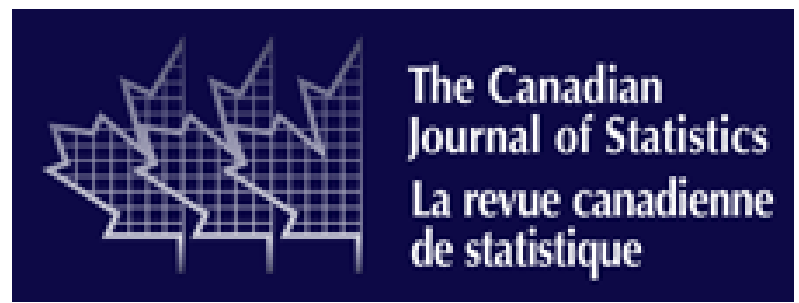

Partially Replicated Two-Level Fractional Factorial Designs

Author(s): Chen-Tuo Liao and Feng-Shun Chai

Source: The Canadian Journal of Statistics / La Revue Canadienne de Statistique, Vol. 32, No. 4 (Dec., 2004), pp. 421-438

Published by: Statistical Society of Canada

Stable URL: http://www.jstor.org/stable/3316025

Accessed: $17 / 11 / 2009$ 05:18

Your use of the JSTOR archive indicates your acceptance of JSTOR's Terms and Conditions of Use, available at http://www.jstor.org/page/info/about/policies/terms.jsp. JSTOR's Terms and Conditions of Use provides, in part, that unless you have obtained prior permission, you may not download an entire issue of a journal or multiple copies of articles, and you may use content in the JSTOR archive only for your personal, non-commercial use.

Please contact the publisher regarding any further use of this work. Publisher contact information may be obtained at http://www.jstor.org/action/showPublisher?publisherCode=ssc.

Each copy of any part of a JSTOR transmission must contain the same copyright notice that appears on the screen or printed page of such transmission.

JSTOR is a not-for-profit service that helps scholars, researchers, and students discover, use, and build upon a wide range of content in a trusted digital archive. We use information technology and tools to increase productivity and facilitate new forms of scholarship. For more information about JSTOR, please contact support@ jstor.org. 
La revue canadienne de statistique

\title{
Partially replicated two-level fractional factorial designs
}

\author{
Chen-Tuo LIAO and Feng-Shun CHAI
}

Key words and phrases: Dispersion effect; lack-of-fit test; parallel flats design; pure error.

MSC 2000: Primary 62K10; secondary 62K05.

Abstract: In a two-level factorial experiment, the authors consider designs with partial duplication which permit estimation of the constant term, all main effects and some specified two-factor interactions, assuming that the other effects are negligible. They construct parallel-flats designs with two identical parallel flats that meet prior specifications; they also consider classes of 3-flat and 4-flat designs. They show that the designs obtained can have a very simple covariance structure and high D-efficiency. They give an algorithm from which they generate a series of practical designs with run sizes $12,16,24$, and 32 .

\section{Plans factoriels fractionnaires en réplique partielle à deux modalités}

Résumé : Dans le cadre d'une expérience factorielle à deux modalités, les auteurs s'intéressent à des plans en réplique partielle permettant l'estimation du terme constant, de tous les effets principaux et de certaines interactions doubles présélectionnées, sous l'hypothèse que les autres interactions sont négligeables. Ils construisent des plans à deux plateaux parallèles identiques rencontrant des spécifications prétablies; leur attention se porte aussi sur les plans à 3 et à 4 plateaux. Ils montrent que les plans ainssi obtenus peuvent avoir une structure de covariance très simple et une haute D-efficacité. Ils fournissent un algorithme grâce auquel ils génèrent une série de plans pratiques pour des essais de tailles 12, 16, 24 et 32 .

\section{INTRODUCTION}

During the initial stages of experimentation, unreplicated two-level fractional factorial designs are widely used to estimate a specified set of factorial effects. The effects of interest are often specified on the basis of knowledge of the investigator and some practical principles of experimentation. See Wu \& Hamada (2000). Since each treatment combination is run at most once in the experiment, it is customary to obtain an experimental error estimate either from the remaining degrees of freedom after fitting the specified effects, or by pooling the small effects identified using normal plotting. In many situations, the investigator may not be sure whether this biased error estimate is reliable for a lack-of-fit test of the model. Therefore, it would be desirable to construct an economical two-level factorial design with some duplicated treatment combinations so as to meet the requirement that pure error be estimable, subject to relatively little loss of efficiency in estimating the specified effects.

Dykstra (1959) presents some two-level factorial designs with partial duplication constructed from combining a full factorial or a fraction of resolution $\mathrm{V}$ or higher with a fraction of resolution III. Most of these designs are not practical due to their large run sizes. An example is given in Pigeon \& McAllister (1989) to show that it is sometimes possible to include partial replication of the design so as to allow for estimation of pure error without sacrificing the orthogonality of the main effects. Their design is actually one composed of three different fractions belonging to a family of regular $2^{n-k}$ fractional factorial designs with the same alias relations and a duplicate of one of the three fractions. It will be shown later that this design is a particular case of parallel flats designs. This motivates us to develop a systematic method of constructing parallel flats designs involving partial duplication.

The construction of orthogonal designs for any set of effects based on the class of parallel flats designs can be found in Srivastava \& Li (1966) and Liao, Iyer \& Vecchia (1996). Chai \& 
Liao (2001) investigate the construction of nonorthogonal three-parallel flats designs (abbreviated as 3-PFDs) of user-specified resolution. In this paper, we focus on the designs that can provide an estimate of pure error and simultaneously estimate any specified set of effects. In the next section, we introduce the notation used in the article, review the definition of parallel flats designs and discuss the information matrix using a parallel flats design. Section 3 discusses the properties of parallel flats designs with two identical parallel flats and presents a set of necessary and sufficient conditions for 3-flat and 4-flat designs to be nonsingular for any given specified factorial effects. Also an algorithm is provided. Finally, Section 4 gives a series of practical designs with run sizes $N=12,16,24,32$ and compares their D-efficiency with the computer-aided designs generated from the DETMAX algorithm of Mitchell (1974).

\section{PRELIMINARIES}

Let $F_{1}, \ldots, F_{n}$ denote the $n$ two-level factors. As is common practice, $F_{1}, \ldots, F_{n}$ will also represent the main effects. The expression $F_{1}^{e_{1}} \ldots F_{n}^{e_{n}}$ will represent a general factorial effect with $e_{i}$ being 0 or 1 . If $e_{i}$ is 1 , then $F_{i}$ appears in the factorial effect, otherwise it does not. The vector $\boldsymbol{e}=\left(e_{1}, \ldots, e_{n}\right)$ (a row vector) is called the defining vector for the general factorial effect $F_{1}^{e_{1}} \cdots F_{n}^{e_{n}}$. The defining vector $e=(0, \ldots, 0)$ is for the constant term $\mu$. We will sometimes use defining vectors to represent factorial effects for convenience. Also let the low level and the high level of a factor be denoted by 0 and 1 , respectively. Then a treatment combination or run is represented by an $n$-tuple whose entries are 0 or 1 . We now review the definition of parallel flats designs. The term parallel flats design will be abbreviated as PFD in the following text.

\subsection{Parallel flats designs.}

A full two-level factorial design with $n$ factors contains all possible $2^{n}$ level combinations, and a regular $2^{n-k}$ fractional factorial contains a subset of these runs, such that the runs of the design satisfy some aliasing relations expressed as linear equations over the Galois Field of order 2, $\mathrm{GF}[2]$. Using geometric terminology, such designs have been called single flat designs.

Hence, a single flat $2^{n-k}$ fractional factorial design $\mathcal{D}_{i}$, defined by the alias matrix $\boldsymbol{A}$ and the coset indicator vector $c_{i}$, consists of all treatment combinations $t=\left(t_{1}, \ldots, t_{n}\right)^{\top}$ satisfying equation $\boldsymbol{A t}=c_{i}$ over GF[2], where $\boldsymbol{A}$ is a $k \times n$ matrix of rank $k, t_{j}$ represents the level of factor $j$ and $c_{i}$ is any $k \times 1$ vector over GF[2]. Moreover, suppose that single flat designs $\mathcal{D}_{1}, \ldots, \mathcal{D}_{f}$ are defined by the same alias matrix $\boldsymbol{A}$ and the coset indicator vectors $c_{1}, \ldots, c_{f}$, respectively. Note that $c_{i}$ are not necessarily distinct. Then the union of all the treatment combinations of these $f$ single flat designs forms an $f$-parallel flats design, denoted by $f$-PFD. Clearly, an $f$-PFD consists of $N=f \times 2^{n-k}$ treatment combinations. Let $C$ be the $k \times f$ coset indicator matrix whose columns are $c_{1}, \ldots, c_{f}$. Thus an $f$-PFD is determined by the pair of matrices $(\boldsymbol{A}, \boldsymbol{C})$.

Parallel flats designs with $n-k$ smaller than $k$ may be more conveniently expressed in the following alternative form. Suppose $B$ is any $n \times(n-k)$ matrix of rank $n-k$ over GF[2] such that $\boldsymbol{A B}=\mathbf{0}$, where $\mathbf{0}$ is a matrix with all elements equal to 0 over GF[2]. Also let $\boldsymbol{z}_{i}=\left(z_{i 1}, \ldots, z_{i n}\right)^{\top}$ be a particular solution to $\boldsymbol{A} \boldsymbol{t}=\boldsymbol{c}_{i}$. Then $\mathcal{D}_{i}$ consists of all $\boldsymbol{t}$ satisfying $\boldsymbol{t}=\boldsymbol{z}_{i}+\boldsymbol{B} \boldsymbol{v}$, where $\boldsymbol{v}$ ranges over all possible vectors of length $n-k$ over GF[2] (there are $2^{n-k}$ such vectors). Hence an $f$-PFD can also be determined by the pair of matrices $(B, Z)$, where $Z$ is the $n \times f$ matrix whose columns are $z_{1}, \ldots, z_{f}$. Also, the $z_{i}$ are not necessarily distinct. The following example illustrates such a design. 
Example 1. Consider the following 16-run design presented in Pigeon \& McAllister (1989).

Factors

\begin{tabular}{cccccccc}
\hline Run & $F_{1}$ & $F_{2}$ & $F_{3}$ & $F_{4}$ & $F_{5}$ & $F_{6}$ & $F_{7}$ \\
\hline 1 & 0 & 0 & 0 & 0 & 0 & 1 & 1 \\
2 & 0 & 1 & 1 & 0 & 1 & 1 & 0 \\
3 & 0 & 0 & 0 & 1 & 1 & 0 & 0 \\
4 & 0 & 1 & 1 & 1 & 0 & 0 & 1 \\
\hline 5 & 1 & 0 & 1 & 0 & 0 & 0 & 0 \\
6 & 1 & 1 & 0 & 0 & 1 & 0 & 1 \\
7 & 1 & 0 & 1 & 1 & 1 & 1 & 1 \\
8 & 1 & 1 & 0 & 1 & 0 & 1 & 0 \\
\hline 9 & 0 & 0 & 0 & 0 & 0 & 1 & 1 \\
10 & 0 & 1 & 1 & 0 & 1 & 1 & 0 \\
11 & 0 & 0 & 0 & 1 & 1 & 0 & 0 \\
12 & 0 & 1 & 1 & 1 & 0 & 0 & 1 \\
\hline 13 & 1 & 1 & 0 & 1 & 1 & 1 & 1 \\
14 & 1 & 0 & 1 & 1 & 0 & 1 & 0 \\
15 & 1 & 1 & 0 & 0 & 0 & 0 & 0 \\
16 & 1 & 0 & 1 & 0 & 1 & 0 & 1 \\
\hline
\end{tabular}

It can easily be checked that each of the sets of four runs $1-4,5-8,9-12$ and 13-16 forms a regular $2^{7-5}$ fractional factorial design in which alias relations are all identical. Namely, they are respectively determined by equations $\boldsymbol{A t}=\boldsymbol{c}_{i}, i=1,2,3,4$, where

$$
\boldsymbol{A}=\left(\begin{array}{lllllll}
1 & 0 & 0 & 0 & 0 & 0 & 0 \\
0 & 1 & 0 & 0 & 0 & 1 & 1 \\
0 & 0 & 1 & 0 & 0 & 1 & 1 \\
0 & 0 & 0 & 1 & 0 & 1 & 0 \\
0 & 0 & 0 & 0 & 1 & 0 & 1
\end{array}\right), \quad \boldsymbol{C}=\left(c_{1}, c_{2}, c_{3}, c_{4}\right)=\left(\begin{array}{llll}
0 & 1 & 0 & 1 \\
0 & 0 & 0 & 1 \\
0 & 1 & 0 & 0 \\
1 & 0 & 1 & 0 \\
1 & 0 & 1 & 0
\end{array}\right)
$$

As a result, this 16-run design is a 4-PFD with two identical flats due to $c_{1}=c_{3}$, i.e., runs $1-4$ are duplicates of runs 9-12. Moreover, it can also be determined by the following two matrices.

$$
\boldsymbol{B}=\left(\begin{array}{cc}
0 & 0 \\
0 & 1 \\
0 & 1 \\
1 & 1 \\
1 & 0 \\
1 & 1 \\
1 & 0
\end{array}\right), \quad \boldsymbol{Z}=\left(\boldsymbol{z}_{1}, \boldsymbol{z}_{2}, \boldsymbol{z}_{3}, \boldsymbol{z}_{4}\right)=\left(\begin{array}{cccc}
0 & 1 & 0 & 1 \\
0 & 0 & 0 & 1 \\
0 & 1 & 0 & 0 \\
1 & 0 & 1 & 0 \\
1 & 0 & 1 & 0 \\
0 & 0 & 0 & 0 \\
0 & 0 & 0 & 0
\end{array}\right) .
$$




\subsection{Linear models and nonsingular designs.}

Let $\boldsymbol{\beta}$ denote the vector of factorial effects that are assumed to be nonzero. The corresponding linear model for the observations from an experiment using a design $\mathcal{D}$ may be written in the form

$$
\boldsymbol{y}=\boldsymbol{X} \boldsymbol{\beta}+\boldsymbol{\varepsilon},
$$

where the matrix $\boldsymbol{X}$ depends on the design $\mathcal{D}$ and is often called the design matrix. Notice that we will use $\boldsymbol{\beta}$ throughout this paper both for the set of effects to be estimated and for the vector of coefficients corresponding to these effects. The vector $\varepsilon$ consists of noise random variables which are assumed to be pairwise uncorrelated with common mean 0 and variance $\sigma^{2}$. $\mathcal{D}$ is said to be nonsingular for $\boldsymbol{\beta}$ if the information matrix $\boldsymbol{X}^{\top} \boldsymbol{X}$ is nonsingular. Best linear unbiased estimates (BLUEs) of all the factorial effects of $\boldsymbol{\beta}$ are available when $\mathcal{D}$ is nonsingular. Particularly, the design is called orthogonal for $\boldsymbol{\beta}$ if $\boldsymbol{X}^{\top} \boldsymbol{X}$ is a diagonal matrix. It is well known that orthogonal designs are universally optimal (A-optimal, D-optimal, E-optimal) for estimating $\boldsymbol{\beta}$ in the class of designs with run size $N$, provided that all the factors have two levels and the constant term $\mu$ is included. But orthogonal designs may not exist for every run size $N$.

\subsection{Information matrix of a parallel flats design.}

Let $\mathcal{D}$ be an $f$-PFD determined by the pair of matrices $(B, Z)$ and suppose the linear model in (2) holds. Let $e_{1}$ and $e_{2}$ be the two defining vectors corresponding to two different elements of $\boldsymbol{\beta}$ and $m\left(\boldsymbol{e}_{1}, \boldsymbol{e}_{2}\right)$ be their corresponding element in the information matrix $\boldsymbol{M}=\boldsymbol{X}^{\top} \boldsymbol{X}$ using $\mathcal{D}$. By Liao, Iyer \& Vecchia (1996), we have

$$
m\left(\boldsymbol{e}_{1}, \boldsymbol{e}_{2}\right)=\left\{\sum_{i=1}^{f}(-1)^{\left(\boldsymbol{e}_{1}+\boldsymbol{e}_{2}\right) \boldsymbol{z}_{i}}\right\}\left\{\sum_{\boldsymbol{v}}(-1)^{\left(\boldsymbol{e}_{1}+\boldsymbol{e}_{2}\right) \boldsymbol{B} v}\right\} .
$$

The exponents of -1 in both factors of (3) are computed using arithmetic modulo 2, but the sums of the powers of -1 are not performed modulo 2 . Recall that $v$ ranges over all possible vectors of length $n-k$ over GF[2].

If $e_{1} B=e_{2} B$, i.e., $\left(e_{1}+e_{2}\right) B=0$ (a zero row vector), then $e_{1}$ and $e_{2}$ are said to be in the same alias set. For a given $B$ of rank $n-k$, there are at most $2^{n-k}$ distinct alias sets defined. On the other hand, suppose $e_{1}$ and $e_{2}$ are in two distinct alias sets, i.e., $\left(e_{1}+e_{2}\right) B \neq 0$, then $\left(e_{1}+e_{2}\right) B v$ consists of equal occurrences of 0 and 1 . Hence, the second term on the right-hand side of (3) is precisely 0 , so that $m\left(e_{1}, e_{2}\right)=0$. This implies that the information matrix can always be expressed as a block diagonal matrix given by

$$
\boldsymbol{M}=\left(\begin{array}{cccc}
\boldsymbol{M}_{1} & \mathbf{0} & \ldots & \mathbf{0} \\
\mathbf{0} & \boldsymbol{M}_{2} & \ldots & \mathbf{0} \\
\vdots & \vdots & \ddots & \vdots \\
\mathbf{0} & \mathbf{0} & \ldots & \boldsymbol{M}_{g}
\end{array}\right)
$$

where each submatrix $\boldsymbol{M}_{j}$ corresponds to an alias set, $j=1, \ldots, g$, and all of the off-diagonal submatrices are zero matrices. Here $g$ is the number of distinct alias sets defined by $\boldsymbol{\beta}$ and $\boldsymbol{B}$.

As mentioned earlier, Pigeon \& McAllister (1989) claim that the design of Example 1 is orthogonal for $\boldsymbol{\beta}=\left\{\mu ; F_{1}, F_{2}, F_{3}, F_{4}, F_{5}, F_{6}, F_{7}\right\}$. Let $e_{1}=(0,1,0,0,0,0,0)$ and $e_{2}=$ $(0,0,1,0,0,0,0)$ which are the defining vectors of $F_{2}$ and $F_{3}$, respectively. Then we have $e_{1} B=e_{2} B=(0,1)$, where $B$ is displayed in (1). So $F_{2}$ and $F_{3}$ are in the same alias set $\left\{F_{2}, F_{3}\right\}$. Similarly, we have another three alias sets $\left\{\mu, F_{1}\right\},\left\{F_{4}, F_{6}\right\}$ and $\left\{F_{5}, F_{7}\right\}$. Moreover, let $e=e_{1}+e_{2}=(0,1,1,0,0,0,0)$, then $e Z=(0,1,0,1)$, where $Z$ is displayed in (1). By Equation (3), the submatrix $M_{j}$ corresponding to this alias set is $16 I_{2}$, where $I_{2}$ is the identity matrix of order 2 . Similarly, the submatrices corresponding to the remaining three alias sets are all equal to $16 I_{2}$. Therefore, the information matrix $M=16 I_{8}$ and the design is orthogonal for 
the $\beta$. Notice that the orthogonality is not universal, as it depends upon the design used and the specification of $\boldsymbol{\beta}$.

\section{PARTIALLY REPLICATED PARALLEL FLATS DESIGNS}

We primarily consider $f$-PFDs with two identical parallel flats. Hereafter we refer to this class of $f$-PFDs as $f$-PFDRs where $f \geq 2$. Among all possible $f$-PFDRs, this paper considers the problem of finding the ones which are D-optimal or high in D-efficiency. Under the restriction that all effects in $\boldsymbol{\beta}$ are estimable (i.e., have unbiased estimators), what is the maximum number of elements in $\beta$ for an $f$-PFDRs with given run size $N=f \cdot 2^{n-k}$ ?

PROPOSITION 1. Let the number of elements in $\beta$ be denoted by $v$ and an $f$-PFDR be determined by the pair of matrices $(B, Z)$, where $B$ is of size $n \times(n-k)$ and rank $n-k$. Then

(a) $v \leq(f-1) 2^{n-k}=(f-1) N / f$ provided that the $f-P F D R$ is nonsingular for $\beta$.

(b) $v \leq f 2^{n-k-1}=N / 2$ provided that the $f-P F D R$ is orthogonal for $\beta$.

(c) For odd $f, v \leq 2^{n-k}=N / f$ provided that the $f$-PFDR is orthogonal for $\beta$.

Proof. To prove statement (a), note that there are $f-1$ distinct parallel flats in an $f$-PFDR and each single flat has $2^{n-k}$ runs. Hence, $v \leq(f-1) 2^{n-k}=(f-1) N / f$ by counting the degrees of freedom for $\boldsymbol{\beta}$.

For statement (b), we consider the case $\boldsymbol{X}^{\top} \boldsymbol{X}=N \boldsymbol{I}_{v}$. Notice that the design matrix $\boldsymbol{X}$ consists of \pm 1 , not 0 and 1 , and $\boldsymbol{\beta}$ contains the constant term $\mu$. Without loss of generality, let

$$
\boldsymbol{X}=\left(\begin{array}{c}
\boldsymbol{X}_{1} \\
\boldsymbol{a}^{\top} \\
\boldsymbol{a}^{\top}
\end{array}\right)
$$

where $\boldsymbol{a}^{\top}$ denotes some treatment combination with two duplicates. We thus have

$$
N \boldsymbol{I}_{v}=\boldsymbol{X}^{\top} \boldsymbol{X}=\boldsymbol{X}_{1}^{\top} \boldsymbol{X}_{1}+2 a a^{\top} .
$$

The quadratic form

$$
\boldsymbol{a}^{\top}\left(N \boldsymbol{I}_{v}\right) \boldsymbol{a}=\boldsymbol{a}^{\top} \boldsymbol{X}^{\top} \boldsymbol{X} \boldsymbol{a}=\boldsymbol{a}^{\top} \boldsymbol{X}_{1}^{\top} \boldsymbol{X}_{1} \boldsymbol{a}+2 \boldsymbol{a}^{\top} \boldsymbol{a} \boldsymbol{a}^{\top} \boldsymbol{a}
$$

yields $N v=\boldsymbol{a}^{\top} \boldsymbol{X}_{1}^{\top} \boldsymbol{X}_{1} \boldsymbol{a}+2 v^{2}$ due to $\boldsymbol{a}^{\top} \boldsymbol{a}=v$. This proves that $v \leq N / 2$ since $\boldsymbol{X}_{1}^{\top} \boldsymbol{X}_{1}$ is nonnegative definite.

Finally to prove (c), assume that the $f$-PFDR is orthogonal for $\boldsymbol{\beta}$ and $f$ is odd. If two distinct effects $e_{1}$ and $e_{2}$ are in the same alias set,

$$
\sum_{v}(-1)^{\left(\boldsymbol{e}_{1}+\boldsymbol{e}_{2}\right) \boldsymbol{B} \boldsymbol{v}}=2^{n-k} \neq 0 \text { and } \sum_{i=1}^{f}(-1)^{\left(\boldsymbol{e}_{1}+\boldsymbol{e}_{2}\right) \boldsymbol{z}_{i}} \neq 0
$$

when $f$ is odd. Thus $m\left(e_{1}, e_{2}\right) \neq 0$ by Equation (3), which contradicts the assumption of orthogonality. So each alias set can contain at most one element of the $\beta$. The proof follows from the fact that there are at most $2^{n-k}$ alias sets defined.

By (b) of Proposition 1, the design of Example 1 is actually a saturated partially replicated orthogonal main-effect plan.

Obviously, 2-PFDRs are twice replicated regular $2^{n-k}$ fractional factorial designs. Therefore, constructing nonsingular 2-PFDRs for $\beta$ is equivalent to searching regular $2^{n-k}$ fractional factorial designs for $\boldsymbol{\beta}$. Moreover, a nonsingular 2-PFDR for $\boldsymbol{\beta}$ is also orthogonal, resulting in a D-optimal design. A complete search algorithm for the sets of eligible alias relations for any given $\boldsymbol{\beta}$ is available in Franklin \& Bailey (1977) and has been implemented in the popular software SAS (SAS Institute, 2002). We now consider 3-PFDRs and 4-PFDRs. 
THEOREM 2. Let a 3-PFDR $\mathcal{D}$ be determined by the two matrices $(B, Z)$, where $\boldsymbol{Z}=$ $\left(z_{1}, z_{2}, z_{3}\right)$. Without loss of generality, let $z_{2}=z_{3}$. Then $\mathcal{D}$ is nonsingular for $\boldsymbol{\beta}$ if and only if each submatrix $M_{j}$ of the information matrix $M$ has one of the following forms:

(a) $M_{j}$ is a scalar $N=3 \times 2^{n-k}$;

(b) $M_{j}$ is either

$$
\left(\begin{array}{cc}
N & N / 3 \\
N / 3 & N
\end{array}\right) \quad \text { or } \quad\left(\begin{array}{cc}
N & -N / 3 \\
-N / 3 & N
\end{array}\right)
$$

Proof. By Srivastava, Anderson \& Mardekian (1984), a necessary condition for an $f$-PFD to be nonsingular for $\boldsymbol{\beta}$ is that the maximum dimension of each diagonal submatrix $\boldsymbol{M}_{j}$ of the information matrix equals the number of distinct parallel flats. There are exactly two distinct parallel flats in a 3-PFDR. Hence, the dimension for every $\boldsymbol{M}_{j}$ must equal 1 or 2 . It is sufficient to complete the conditional ("if") part of the proof by proving the only possible nonsingular $\boldsymbol{M}_{j}$ are given in (a) and (b).

First, if the dimension of $\boldsymbol{M}_{\boldsymbol{j}}$ is one, then by Equation (3), the only choice for $\boldsymbol{M}_{\boldsymbol{j}}$ is a scalar and equals $N\left(=3 \times 2^{n-k}\right)$. Second, suppose the dimension of $M_{j}$ is two and $e_{1}$ and $e_{2}$ are the two distinct factorial effects in the alias set corresponding to $\boldsymbol{M}_{j}$. If the $\boldsymbol{z}_{i}$ are chosen such that $e \boldsymbol{z}_{i}=\left(\boldsymbol{e}_{1}+\boldsymbol{e}_{2}\right) \boldsymbol{z}_{i}=0$ for all $i$, then by Equation (3) again, $m\left(\boldsymbol{e}_{1}, \boldsymbol{e}_{2}\right)=N$. If $\boldsymbol{e} \boldsymbol{z}_{i}=1$ for all $i$, then $m\left(e_{1}, e_{2}\right)=-N$. For these two cases, $\boldsymbol{M}_{j}$ is singular. For the remaining cases, $\boldsymbol{M}_{j}$ is nonsingular, i.e., the set $\left\{e z_{1}, e z_{2}, e z_{2}\right\}$ has either exactly two 0 's and one 1 , which makes $m\left(e_{1}, e_{2}\right)=N / 3$, or one 0 and two 1 's, which makes $m\left(e_{1}, e_{2}\right)=-N / 3$.

On the other hand, if any $\boldsymbol{M}$ of the block diagonal form with submatrices $\boldsymbol{M}_{j}$ given in (a) or (b) is nonsingular. Then, $\mathcal{D}$ is nonsingular for $\boldsymbol{\beta}$. This completes the proof.

Clearly, the two forms of $\boldsymbol{M}_{j}$ given in (b) of Theorem 2 have equivalent information since their eigenvalues are identical and each eigenvalue also has the same multiplicity, which are $2 N / 3$ and $4 N / 3$ both with multiplicity 1 . So the 3-PFDRs have such a simple covariance structure. Theorem 2 applies to generate 12-run and 24-run designs with 4 and 8 degrees of freedom, respectively, for estimating the variance of pure error in next section.

THEOREM 3. Let a 4-PFDR $\mathcal{D}$ be determined by the pair of matrices $(\boldsymbol{B}, \boldsymbol{Z})$, where $\boldsymbol{Z}=$ $\left(z_{1}, z_{2}, z_{3}, z_{4}\right)$. Without loss of generality, let $z_{3}=z_{4}$. Then $\mathcal{D}$ is nonsingularfor $\beta$ if and only if each submatrix $M_{j}$ of the information matrix $M$ has one of the following forms:

(a) $M_{j}$ is a scalar $N=4 \times 2^{n-k}$;

(b) $M_{j}$ is one of

$$
\left(\begin{array}{cc}
N & 0 \\
0 & N
\end{array}\right), \quad\left(\begin{array}{cc}
N & N / 2 \\
N / 2 & N
\end{array}\right), \quad\left(\begin{array}{cc}
N & -N / 2 \\
-N / 2 & N
\end{array}\right)
$$

(c) $M_{j}$ is, after a suitable permutation of the factorial effects involved, one of

$$
\left(\begin{array}{ccc}
N & 0 & N / 2 \\
0 & N & N / 2 \\
N / 2 & N / 2 & N
\end{array}\right), \quad\left(\begin{array}{ccc}
N & 0 & N / 2 \\
0 & N & -N / 2 \\
N / 2 & -N / 2 & N
\end{array}\right), \quad\left(\begin{array}{ccc}
N & 0 & -N / 2 \\
0 & N & -N / 2 \\
-N / 2 & -N / 2 & N
\end{array}\right) .
$$

Proof. There are exactly three distinct parallel flats in a 4-PFDR. Again by Srivastava, Anderson \& Mardekian (1984), the dimension for every $\boldsymbol{M}_{j}$ must be less than or equal to 3 . We thus need to prove the $M_{j}$ of (a), (b) and (c) are the only possible nonsingular submatrices. 
(a) If the dimension of $M_{j}$ is one, then the $M_{j}$ is a scalar and equals $N\left(=4 \times 2^{n-k}\right)$.

(b) Suppose the dimension of $M_{j}$ is two and $e_{1}$ and $e_{2}$ are the two distinct factorial effects in the alias set corresponding to $\boldsymbol{M}_{j}$. By Equation (3), if $e z_{i}=\left(e_{1}+e_{2}\right) z_{i}=0$ for all $i$, then $m\left(e_{1}, e_{2}\right)=N$. Similarly, if $\boldsymbol{e z}_{i}=1$ for all $i$, then $m\left(\boldsymbol{e}_{1}, \boldsymbol{e}_{2}\right)=-N$. For these two cases, $\boldsymbol{M}_{j}$ is singular. For the remaining cases, $\boldsymbol{M}_{j}$ is nonsingular, i.e., the set $\left\{e z_{1}, e z_{2}, e z_{3}, e z_{3}\right\}$ has exactly two 0 's and two 1's, which makes $m\left(e_{1}, e_{2}\right)=0$; exactly one 1 and three 0 's, which makes $m\left(e_{1}, e_{2}\right)=N / 2$; or one 0 and three 1 's, which makes $m\left(e_{1}, e_{2}\right)=-N / 2$.

(c) Suppose the dimension of $M_{j}$ is three and $e_{1}, e_{2}$ and $e_{3}$ are the three distinct factorial effects in the alias set corresponding to $M_{j}$. Let $e_{1}^{*}=e_{1}+e_{2}, e_{2}^{*}=e_{1}+e_{3}$ and $e_{3}^{*}=e_{2}+e_{3}$. Also let vectors $w_{\ell}=\left(e_{\ell}^{*} z_{1}, e_{\ell}^{*} z_{2}, e_{\ell}^{*} z_{3}, e_{\ell}^{*} z_{3}\right)$ for $\ell=1,2,3 . M_{j}$ is singular if $m\left(\boldsymbol{e}_{\ell}, \boldsymbol{e}_{\ell^{\prime}}\right)=N$ or $-N$ for some $\ell \neq \ell^{\prime}$. So none of $\boldsymbol{w}_{\ell}$ can be $(0,0,0,0)$ or $(1,1,1,1)$. Note that $e_{1}^{*}+e_{2}^{*}+e_{3}^{*}=0$. Hence, the set $\left\{w_{1}, w_{2}, w_{3}\right\}$ must be one of $\{(1,0,0,0),(0,1,0,0),(1,1,0,0)\} ;\{(1,0,0,0),(1,0,1,1),(0,0,1,1)\} ;\{(1,0,1,1)$, $(0,1,1,1),(1,1,0,0)\}$, leading the $\boldsymbol{M}_{j}$ to the forms given in (c).

On the other hand, any $\boldsymbol{M}$ of the block diagonal forms with submatrices $\boldsymbol{M}_{j}$ given in one of (a), (b), (c) is nonsingular, hence, $\mathcal{D}$ is nonsingular for $\boldsymbol{\beta}$. This completes the proof.

Notice that the three choices of $M_{j}$ described in (c) of Theorem 3 have equivalent information. Every $\boldsymbol{M}_{j}$ has the same eigenvalues $N,(1-\sqrt{2} / 2) N$ and $(1+\sqrt{2} / 2) N$ all with multiplicity 1.

The results can be extended to $f$-PFDRs for $f \geq 5$ with more extensive work. The situation becomes complicated for large $f$. For example, there are nine possible nonsingular $\boldsymbol{M}_{j}$ of dimension 3 in the case of 5-PFDRs. We now present below an algorithm for constructing nonsingular 4-PFDRs for any given $\beta$, which can be readily modified for 3-PFDRs. For $f \geq 5$, we have yet to find a practical algorithm.

According to Theorem 3, we simply consider the 4-PFDRs with $Z=\left(z_{1}, z_{2}, \mathbf{0}, \mathbf{0}\right)$. Note that the submatrix $M_{j}$ which is a diagonal matrix in (b) of the theorem is always desired, since it has larger determinant than the rest two submatrices. Furthermore, a 4-PFDR attains the maximum determinant as its information matrix contains maximal number of submatrices $\boldsymbol{M}_{j}$ with the possibly smallest dimension. Therefore, the algorithm makes an attempt to choose (i) $\boldsymbol{B}$ such that each of all $2^{n-k}$ alias sets contains the number of effects as equal as possible; (ii) $z_{1}$ and $z_{2}$ such that all the $\boldsymbol{M}_{\boldsymbol{j}}$ defined by the $\boldsymbol{B}$ are nonsingular and result in maximal number of diagonal $\boldsymbol{M}_{j}$ with dimension 2, i.e., the first matrix in (b) of Theorem 3.

The following procedure shows us how to choose appropriate $B, z_{1}$ and $z_{2}$ to obtain the highest D-efficiency, defined by

$$
D_{e}=\frac{1}{N}\left|\boldsymbol{X}^{\top} \boldsymbol{X}\right|^{1 / v}
$$

within the class of 4-PFDRs for any given $\beta$, where $v$ denotes the number of elements in $\beta . D_{e}$ is the relative efficiency, according to D-optimality criterion, of the underlying design to orthogonal design with run size $N$.

Step 1: Let $m_{j}$ be the number of effects in alias set $G_{j}, j=1, \ldots, 2^{n-k}$. Choose matrix $B$ such that each $m_{j} \leq 3$ and the $m_{j}$ are as nearly equal as possible. A complete search algorithm for eligible $\boldsymbol{B}$ can be obtained by modifying the algorithm of Franklin \& Bailey (1977).

Step 2: Consider those $G_{j}$ with $m_{j}=2$. Let $e_{1 j}, e_{2 j} \in G_{j}$ and $e_{j}^{*}=e_{1 j}+e_{2 j}$. Choose $z_{1}$ and $z_{2}$ such that as many as possible of the sets $\left\{e_{j}^{*} z_{1}, e_{j}^{*} z_{2}\right\}$ have exactly two 1 's. This is so as to have the maximal number of the desired $M_{j}$ (the diagonal one) in (b) of Theorem 3. Notice that one restriction for the choice of $z_{1}$ and $z_{2}$ is that $\left\{e_{j}^{*} z_{1}, e_{j}^{*} z_{2}\right\}$ cannot have two 0 's for any $j$, since this case makes the corresponding $M_{j}$ singular. 
Step 3: Consider those $G_{j}$ with $m_{j}=3$. Let $e_{1 j}, e_{2 j}, e_{3 j} \in G_{j}$ and $e_{1 j}^{*}=e_{1 j}+e_{2 j}$, $e_{2 j}^{*}=e_{1 j}+e_{3 j}, e_{3 j}^{*}=e_{2 j}+e_{3 j}$. Reset $z_{1}, z_{2}$ (already chosen in Step (2)) or both if necessary, such that the set $\left\{\left(e_{1 j}^{*} z_{1}, e_{1 j}^{*} z_{2}\right),\left(e_{2 j}^{*} z_{1}, e_{2 j}^{*} z_{2}\right),\left(e_{3 j}^{*} z_{1}, e_{3 j}^{*} z_{2}\right)\right\}$ is $\{(1,1),(0,1),(1,0)\}$. This ensures that all the corresponding $\boldsymbol{M}_{j}$ of dimension 3 are nonsingular.

The following example is given to illustrate the algorithm.

Example 2. For a $2^{4}$ experiment, suppose $\beta$ consists of the following factorial effects:

$$
\mu ; F_{1}, F_{2}, F_{3}, F_{4} ; F_{1} F_{3}, F_{1} F_{4}, F_{2} F_{3}, F_{2} F_{4}, F_{3} F_{4} \text {. }
$$

In this case, there are ten effects to be estimated. Any factorial effect that is not in the span of the above effects is assumed to be zero. Our goal is to construct a 4-PFDR with $N=16$ for estimating these factorial effects. Following the algorithm, we have

Step 1: Choose

$$
\boldsymbol{B}(4 \times 2)=\left(\begin{array}{ll}
0 & 0 \\
1 & 0 \\
0 & 1 \\
1 & 1
\end{array}\right)
$$

There are four alias sets $G_{1}=\left\{\mu, F_{1}\right\}, G_{2}=\left\{F_{2}, F_{3} F_{4}\right\}, G_{3}=\left\{F_{3}, F_{1} F_{3}, F_{2} F_{4}\right\}$, and $G_{4}=\left\{F_{4}, F_{1} F_{4}, F_{2} F_{3}\right\}$ determined by $B$

Step 2: For $G_{1}, e_{1}^{*}=(0,0,0,0)+(1,0,0,0)=(1,0,0,0)$ and for $G_{2}, e_{2}^{*}=(0,1,0,0)+$ $(0,0,1,1)=(0,1,1,1)$. Hence, choose $z_{1}=(1,1,0,0)^{\top}$ and $z_{2}=(1,1,0,0)^{\top}$ such that $\left\{e_{1}^{*} z_{1}, e_{1}^{*} z_{2}\right\}=\left\{e_{2}^{*} z_{1}, e_{2}^{*} z_{2}\right\}=\{1,1\}$.

Step 3: For $G_{3}$ and $G_{4},\left\{e_{13}^{*}, e_{23}^{*}, e_{33}^{*}\right\}=\left\{e_{14}^{*}, e_{24}^{*}, e_{34}^{*}\right\}=\{(1,0,0,0),(0,1,1,1),(1,1,1,1)\}$. Here, we need to reset $z_{1}$ to be $(1,1,1,0)^{\top}$ such that

$$
\begin{aligned}
& \left\{\left(e_{13}^{*} z_{1}, e_{13}^{*} z_{2}\right),\left(e_{23}^{*} z_{1}, e_{23}^{*} z_{2}\right),\left(e_{33}^{*} z_{1}, e_{33}^{*} z_{2}\right)\right\} \\
& \quad=\left\{\left(e_{14}^{*} z_{1}, e_{14}^{*} z_{2}\right),\left(e_{24}^{*} z_{1}, e_{24}^{*} z_{2}\right),\left(e_{34}^{*} z_{1}, e_{34}^{*} z_{2}\right)\right\}=\{(1,1),(0,1),(1,0)\}
\end{aligned}
$$

Then (also see Section 2.1) the desired nonsingular 16-run 4-PFDR, determined by matrix $\boldsymbol{B}$ of Step (1) and

$$
\boldsymbol{Z}(4 \times 4)=\left(\boldsymbol{z}_{1}, \boldsymbol{z}_{2}, \mathbf{0}, \mathbf{0}\right)=\left(\begin{array}{cccc}
1 & 1 & 0 & 0 \\
1 & 1 & 0 & 0 \\
1 & 0 & 0 & 0 \\
0 & 0 & 0 & 0
\end{array}\right)
$$

for $\boldsymbol{\beta}$ is $\left\{(1,1,1,0)^{\top},(1,1,0,1)^{\top},(1,0,1,1)^{\top},(1,0,0,0)^{\top},(1,1,0,0)^{\top},(1,1,1,1)^{\top}\right.$, $(1,0,0,1)^{\top},(1,0,1,0)^{\top},(0,0,0,0)^{\top},(0,0,1,1)^{\top},(0,1,0,1)^{\top},(0,1,1,0)^{\top},(0,0,0,0)^{\top}$, $\left.(0,0,1,1)^{\top},(0,1,0,1)^{\top},(0,1,1,0)^{\top}\right\}$. Its information matrix is given by

$$
\boldsymbol{M}=\left(\begin{array}{cccc}
M_{1} & \mathbf{0} & \mathbf{0} & \mathbf{0} \\
\mathbf{0} & M_{2} & \mathbf{0} & \mathbf{0} \\
\mathbf{0} & \mathbf{0} & M_{3} & \mathbf{0} \\
\mathbf{0} & \mathbf{0} & \mathbf{0} & M_{4}
\end{array}\right)
$$


where $M_{1}=M_{2}=16 I_{2}$ and

$$
\boldsymbol{M}_{3}=\boldsymbol{M}_{4}=\left(\begin{array}{ccc}
16 & 0 & -8 \\
0 & 16 & -8 \\
-8 & -8 & 16
\end{array}\right)
$$

\section{PARTIALLY REPLICATED DESIGNS OF USER-SPECIFIED RESOLUTION}

In this section, we report a series of 12-run 3-PFDRs, 16-run 4-PFDRs, 24-run 3-PFDRs and 32-run 4-PFDRs for two classes of specified factorial effects. These two classes of specified factorial effects under consideration, also suggested by Srivastava \& Li (1996), are of practical interest. We now describe them as follows. Suppose that $n$ factors are divided into $p$ groups and the $i$ th group has $n_{i}$ factors, $1 \leq i \leq p$. Let $p^{\prime}$ be the number of these groups that are of interest. That implies we consider only the two-factor interactions involving the factors of the $p^{\prime}$ groups of interest. Without loss of generality, we always choose the first $p^{\prime}$ groups among $p$ groups and discuss only two cases $p^{\prime}=p$ and $p^{\prime}=p-1$. The first class of specified effects, called Class I: interactions within groups, consists of the constant term, all main effects and all twofactor interactions within each of the $p^{\prime}$ groups of interest. Class II of the specified effects, called interactions between groups, consists of the constant term, all main effects and all two-factor interactions between $p^{\prime}$ groups of interest.

To simplify the presentation of the designs obtained, we write each column of matrices $B$ and $\boldsymbol{Z}$ by listing the position numbers of nonzero entries. For example, the $\boldsymbol{B}$ of (5) is written as $\boldsymbol{B}=\{24,34\}$. Recall that we set $\boldsymbol{z}_{f-1}=\boldsymbol{z}_{f}=\mathbf{0}$ for $f$-PFDRs in the algorithm. Hence, we only write out the first $f-2$ columns of $\boldsymbol{Z}$. For example, the $\boldsymbol{Z}$ of (6) is written as $\boldsymbol{Z}=\{123,12\}$. Also write $\boldsymbol{\beta}=\left\{\mu ; F_{1}, \ldots, F_{n} ; F_{1} F_{2}, \ldots, F_{i} F_{j}\right\}$ as $\boldsymbol{\beta}=\{0 ; 1, \ldots, n ; 1 \cdot 2, \ldots, i \cdot j\}$. For example, $\boldsymbol{\beta}=\left\{\mu ; F_{1}, F_{2}, F_{3} ; F_{1} F_{2}, F_{1} F_{3}\right\}=\{0 ; 1,2,3 ; 1 \cdot 2,1 \cdot 3\}$. In the following, we recall some notation needed in the coming tables.

$n=$ number of factors to be assessed;

$p=$ number of groups;

$p^{\prime}=$ number of groups actually involved in the two-factor interactions;

$n_{i}=$ number of factors in the $i$ th $(i=1, \ldots, p)$ group;

$v=$ number of the nonzero effects to be estimated.

Note that a design is nonsingular for a specified set of effects; it is also nonsingular for any subset of the specified set of effects. Thus the designs presented in the following tables can be applicable to more situations. Although we restrict $p^{\prime}$ to be $p$ or $p-1$, we do not present all possible cases in Tables $1,4,7,10$ so as to keep them a reasonable size for presentation. Of course, one can always use the algorithm in Section 3 to construct their desired design which is not listed in the tables. To use the tables, an experimenter will proceed as follows. Identify $n$ the number of factors to be assessed and the number of runs $N=12,16,24$, or 32 to be considered for the experiment. Specify a set of two-factor interactions which are to be estimated. Then check the tables and pick up the case (design) in which the corresponding column of "Estimable interactions" includes the specified two-factor interactions.

\subsection{2-run 3-PFDRs and 16-run 4-PFDRs.}

Table 1 identifies the cases for which we can construct 16-run 4-PFDRs and, in some cases, 12 run 3-PFDRs for Class I specifications. It reports the two-factor interactions to be estimated; in addition, all $n$ main effects and the constant term are to be estimated. 
TABLE 1: List of specified sets of within groups two-factor interactions for which there are 12-run 3-PFDRs or 16-run 4-PFDRs.

\begin{tabular}{rrrrccc}
\hline Case & $n$ & $v$ & $p$ & $p^{\prime}$ & $\left(n_{1}, \ldots, n_{p}\right)$ & Estimable interactions \\
\hline 1 & 4 & 8 & 2 & 1 & $(3,1)$ & $\{1,2,3\} \times\{1,2,3\}$ \\
2 & 4 & 11 & 1 & 1 & $(4)$ & $\{1,2,3,4\} \times\{1,2,3,4\}$ \\
3 & 5 & 10 & 2 & 2 & $(3,2)$ & $\{1,2,3\} \times\{1,2,3\} \cup\{4 \cdot 5\}$ \\
4 & 5 & 12 & 2 & 1 & $(4,1)$ & $\{1,2,3,4\} \times\{1,2,3,4\}$ \\
5 & 6 & 8 & 2 & 1 & $(2,4)$ & $\{1 \cdot 2\}$ \\
6 & 6 & 9 & 3 & 2 & $(2,2,2)$ & $\{1 \cdot 2,3 \cdot 4\}$ \\
7 & 6 & 10 & 2 & 1 & $(3,3)$ & $\{1,2,3\} \times\{1,2,3\}$ \\
8 & 6 & 10 & 3 & 3 & $(2,2,2)$ & $\{1 \cdot 2,3 \cdot 4,5 \cdot 6\}$ \\
9 & 6 & 11 & 3 & 2 & $(3,2,1)$ & $\{1,2,3\} \times\{1,2,3\} \cup\{4 \cdot 5\}$ \\
10 & 7 & 8 & 1 & 0 & $(7)$ & None \\
11 & 7 & 9 & 2 & 1 & $(2,5)$ & $\{1 \cdot 2\}$ \\
12 & 7 & 10 & 3 & 2 & $(2,2,3)$ & $\{1 \cdot 2,3 \cdot 4\}$ \\
13 & 7 & 11 & 4 & 3 & $(2,2,2,1)$ & $\{1 \cdot 2,3 \cdot 4,5 \cdot 6\}$ \\
14 & 7 & 11 & 2 & 1 & $(3,4)$ & $\{1,2,3\} \times\{1,2,3\}$ \\
15 & 8 & 10 & 2 & 1 & $(2,6)$ & $\{1 \cdot 2\}$ \\
16 & 8 & 11 & 3 & 2 & $(2,2,4)$ & $\{1 \cdot 2,3 \cdot 4\}$ \\
17 & 8 & 12 & 4 & 3 & $(2,2,2,2)$ & $\{1 \cdot 2,3 \cdot 4,5 \cdot 6\}$ \\
18 & 8 & 12 & 2 & 1 & $(3,5)$ & $\{1,2,3\} \times\{1,2,3\}$ \\
19 & 9 & 11 & 2 & 1 & $(2,7)$ & $\{1 \cdot 2\}$ \\
20 & 9 & 12 & 3 & 2 & $(2,2,5)$ & $\{1 \cdot 2,3 \cdot 4\}$ \\
\hline & & & & &
\end{tabular}

We will explain Case 1 of Tables 1 and 2 in detail; all the other cases in Tables 3-12 follow similarly. For Case 1 of Table 1, we are interested in estimating the constant term $\mu$, all main effects and all two-factor interactions within the group $\left\{F_{1}, F_{2}, F_{3}\right\}$ for a $2^{4}$ factorial experimental design. Thus we have $n=4, v=8, p=2, p^{\prime}=1,\left(n_{1}, n_{2}\right)=(3,1)$. The defining vectors of $\boldsymbol{\beta}$ can be expressed as $\boldsymbol{\beta}=\{0 ; 1,2,3,4 ; 1 \cdot 2,1 \cdot 3,2 \cdot 3\}$. In Table 1 , we have listed only the second order interactions in $\boldsymbol{\beta}$ since in this line and every line in the tables, all $n$ main effects and the constant term are estimated. Moreover we have adopted the notation that $A \times B$ means all two-factor interactions obtained by selecting one element of $A$ and one of $B$.

In Tables 2 and 3, we list corresponding 12-run 3-PFDRs and 16-run 4-PFDRs which correspond, where possible, to the cases identified in Table 1. We also provide two measures of the D-efficiency of the designs.

TABLE 2: Some 12-run 3-PFDRs corresponding to the possible cases in Table 1 along with their measures of efficiency $D_{e}$ (3-PFDR) and $D_{e}(\mathrm{MD})$.

\begin{tabular}{ccccc}
\hline Case & $\boldsymbol{B}=\left\{\boldsymbol{b}_{1}, \boldsymbol{b}_{2}\right\}$ & $\boldsymbol{Z}=\left\{\boldsymbol{z}_{1}\right\}$ & $D_{e}(3-\mathrm{PFDR})$ & $D_{e}(\mathrm{MD})$ \\
\hline 1 & $\{34,24\}$ & $\{1234\}$ & 0.943 & 0.943 \\
5 & $\{3456,136\}$ & $\{1246\}$ & 0.943 & 0.943 \\
10 & $\{1346,2356\}$ & $\{1237\}$ & 0.943 & 0.943 \\
\hline
\end{tabular}


To the best of our knowledge, for any given $\beta$, there is no systematic construction method available in the literature for optimal partially replicated designs. However, a very natural method is to augment an optimal design for $\boldsymbol{\beta}$ with some duplicates of its fraction. Therefore, in Tables 2 and 3, we compare the D-efficiency of the 12-run 3-PFDRs and 16-run 4-PFDRs we have obtained with that of the designs generated by the following approach. For a given $\beta$, we first generate a "D-optimal" design by the OPTEX procedure (GENERATE CRITERION=D METHOD=DETMAX ITER=100 $\mathrm{N}=8$ (12)) of SAS/QC software (SAS Institute 2002). Here we choose the DETMAX algorithm of Mitchell (1974) for searching the D-optimal design with $N=8$ and $N=12$, respectively, using 100 iterations. Then we augment the generated D-optimal design with four more runs chosen from its treatment combinations. There are $\left(\begin{array}{l}8 \\ 4\end{array}\right)=105$ and $\left(\begin{array}{c}12 \\ 4\end{array}\right)=495$ possible choices for the D-optimal design with $N=8$ and $N=12$, respectively. We finally select the augmented designs of size $N=12$ and $N=16$ with the highest D-efficiency to be compared with our designs.

TABLE 3: Some 16-run 4-PFDRs corresponding to the cases in Table 1 along with their efficiency measures $D_{e}(4-\mathrm{PFDR})$ and $D_{e}(\mathrm{MD})$.

\begin{tabular}{ccccc}
\hline Case & $\boldsymbol{B}=\left\{\boldsymbol{b}_{1}, \boldsymbol{b}_{2}\right\}$ & $\boldsymbol{Z}=\left\{\boldsymbol{z}_{1}, \boldsymbol{z}_{2}\right\}$ & $D_{e}(4-\mathrm{PFDR})$ & $D_{e}(\mathrm{MD})$ \\
\hline 1 & $\{34,24\}$ & $\{1234,1234\}$ & 1.000 & 1.000 \\
2 & $\{34,24\}$ & $\{123,14\}$ & 0.828 & 0.823 \\
3 & $\{34,24\}$ & $\{12345,14\}$ & 0.871 & 0.890 \\
4 & $\{34,24\}$ & $\{12345,14\}$ & 0.794 & 0.809 \\
5 & $\{3456,136\}$ & $\{1246,1246\}$ & 1.000 & 0.958 \\
6 & $\{3456,136\}$ & $\{1246,1256\}$ & 0.926 & 0.914 \\
7 & $\{3456,136\}$ & $\{1246,2356\}$ & 0.871 & 0.907 \\
8 & $\{1234,1235\}$ & $\{236,36\}$ & 0.812 & 0.871 \\
9 & $\{3456,136\}$ & $\{1246,12\}$ & 0.828 & 0.837 \\
10 & $\{1346,2356\}$ & $\{1237,1237\}$ & 1.000 & 0.968 \\
11 & $\{1346,2356\}$ & $\{1237,4567\}$ & 0.926 & 0.957 \\
12 & $\{1346,2356\}$ & $\{1237,4567\}$ & 0.871 & 0.901 \\
13 & $\{1346,2356\}$ & $\{1237,4567\}$ & 0.828 & 0.862 \\
14 & $\{1346,2356\}$ & $\{1237,4567\}$ & 0.828 & 0.882 \\
15 & $\{1346,2356\}$ & $\{1237,4567\}$ & 0.871 & 0.928 \\
16 & $\{1346,2356\}$ & $\{1237,4567\}$ & 0.828 & 0.882 \\
17 & $\{1346,2356\}$ & $\{1237,4567\}$ & 0.794 & 0.827 \\
18 & $\{1346,2356\}$ & $\{1237,4567\}$ & 0.794 & 0.849 \\
19 & $\{35789,25689\}$ & $\{13569,24789\}$ & 0.828 & 0.907 \\
20 & $\{35789,25689\}$ & $\{13569,24789\}$ & 0.794 & 0.855 \\
\hline
\end{tabular}

By Proposition $1(\mathrm{a}), v \leq(f-1) N / f=(3-1) 12 / 3=8$. Hence we only can generate 12-run 3-PFDRs for Cases 1, 5, and 10 in Table 1; these are listed in Table 2. The notation MD denotes the design obtained by the Mitchell algorithm.

For clarity we spell out the interpretation of the line for Case 1 in Table 2. One possible 3-PFDR for the $\boldsymbol{\beta}$ for Case 1 is the set of treatment combinations $t$ satisfying $t=z_{i}+B \boldsymbol{v}$, for 
$i=1,2,3$, where

$$
\boldsymbol{B}=\left(\boldsymbol{b}_{1}, \boldsymbol{b}_{2}\right)=\left(\begin{array}{cc}
0 & 0 \\
1 & 0 \\
0 & 1 \\
1 & 1
\end{array}\right), \quad \boldsymbol{Z}=\left(\boldsymbol{z}_{1}, \boldsymbol{z}_{2}, \boldsymbol{z}_{3}\right)=\left(\begin{array}{ccc}
1 & 0 & 0 \\
1 & 0 & 0 \\
1 & 0 & 0 \\
1 & 0 & 0
\end{array}\right)
$$

and $\boldsymbol{v}$ ranges over all possible vectors of length 2 over GF[2]. Hence (also see Section 2.1) in Case 1 in Table 2, we use $B=\{24,34\}$ and $Z=\{1234\}$ to represent the desired 12-run 3-PFDR.

Table 3 is analogous to Table 2 but for 16-run designs; it displays the 16-run 4-PFDRs obtained corresponding to the cases in Table 1.

We now turn to the second class of specified interactions of interest-the situation where an experimenter is interested in all two-factor interactions between members of $p^{\prime}$ groups of factors. Factorial effects for these interactions between groups are given in Table 4. The notation matches that in Table 1.

TABLE 4: List of specified sets of between groups two-factor interactions for which there are 12-run 3-PFDRs or 16-run 4-PFDRs.

\begin{tabular}{rrrrccc}
\hline Case & $n$ & $v$ & $p$ & $p^{\prime}$ & $\left(n_{1}, \ldots, n_{p}\right)$ & Estimable interactions \\
\hline 1 & 4 & 8 & 2 & 2 & $(1,3)$ & $\{1\} \times\{2,3,4\}$ \\
2 & 4 & 9 & 2 & 2 & $(2,2)$ & $\{1,2\} \times\{3,4\}$ \\
3 & 5 & 8 & 3 & 2 & $(1,2,2)$ & $\{1\} \times\{2,3\}$ \\
4 & 5 & 9 & 3 & 2 & $(1,3,1)$ & $\{1\} \times\{2,3,4\}$ \\
5 & 5 & 10 & 2 & 2 & $(1,4)$ & $\{1\} \times\{2,3,4,5\}$ \\
6 & 5 & 10 & 3 & 2 & $(2,2,1)$ & $\{1,2\} \times\{3,4\}$ \\
7 & 5 & 12 & 2 & 2 & $(2,3)$ & $\{1,2\} \times\{3,4,5\}$ \\
8 & 6 & 9 & 3 & 2 & $(1,2,3)$ & $\{1\} \times\{2,3\}$ \\
9 & 6 & 10 & 3 & 2 & $(1,3,2)$ & $\{1\} \times\{2,3,4\}$ \\
10 & 6 & 11 & 3 & 2 & $(1,4,1)$ & $\{1\} \times\{2,3,4,5\}$ \\
11 & 6 & 12 & 2 & 2 & $(1,5)$ & $\{1\} \times\{2,3,4,5,6\}$ \\
12 & 6 & 11 & 3 & 2 & $(2,2,2)$ & $\{1,2\} \times\{3,4\}$ \\
13 & 7 & 10 & 3 & 2 & $(1,2,4)$ & $\{1\} \times\{2,3\}$ \\
14 & 7 & 11 & 3 & 2 & $(1,3,3)$ & $\{1\} \times\{2,3,4\}$ \\
15 & 7 & 12 & 3 & 2 & $(1,4,2)$ & $\{1\} \times\{2,3,4,5\}$ \\
16 & 7 & 12 & 3 & 2 & $(2,2,3)$ & $\{1,2\} \times\{3,4\}$ \\
17 & 8 & 11 & 3 & 2 & $(1,2,5)$ & $\{1\} \times\{2,3\}$ \\
18 & 8 & 12 & 3 & 2 & $(1,3,4)$ & $\{1\} \times\{2,3,4\}$ \\
19 & 9 & 12 & 3 & 2 & $(1,2,6)$ & $\{1\} \times\{2,3\}$ \\
20 & 10 & 12 & 3 & 2 & $(1,1,8)$ & $\{1 \cdot 2\}$ \\
\hline & & & & & & \\
\hline
\end{tabular}

By Proposition 1(a), we generate 12-run 3-PFDRs only for Cases 1 and 3; these designs are displayed in Table 5 along with the measures of efficiency $D_{e}(3-\mathrm{PFDR})$ and $D_{e}(\mathrm{MD})$. 
TABLE 5: Some 12-run 3-PFDRs corresponding to the possible cases in Table 4 along with their measures of efficiency $D_{e}$ (3-PFDR) and $D_{e}(\mathrm{MD})$.

\begin{tabular}{lcccc}
\hline Case & $\boldsymbol{B}=\left\{\boldsymbol{b}_{1}, \boldsymbol{b}_{2}\right\}$ & $\boldsymbol{Z}=\left\{\boldsymbol{z}_{1}\right\}$ & $D_{e}(3-\mathrm{PFDR})$ & $D_{e}(\mathrm{MD})$ \\
\hline 1 & $\{34,24\}$ & $\{1234\}$ & 0.943 & 0.943 \\
3 & $\{245,1235\}$ & $\{124\}$ & 0.943 & 0.943 \\
\hline
\end{tabular}

The corresponding results for 16-run 4-PFDRs are displayed in Table 6.

As discussed in Galil \& Kiefer (1980) and Welch (1984), the DETMAX algorithm of Mitchell is a powerful heuristic and is very successful in finding D-optimal designs. Compared with the designs generated from Mitchell's algorithm, the D-efficiency of most of the PFDRs we obtain is equal to or close to that of the corresponding Mitchell designs. It is interesting to observe that the PFDRs obtained are even better for some cases (check Cases 2, 5, 6, 10 of Table 3 and Case 3 of Table 6). Moreover, all the orthogonal 16-run 4-PFDRs obtained (i.e., those with $D_{e}(4-\mathrm{PFDR})=$ 1.000) turn out to be 16-run 2-PFDRs (with $z_{1}=z_{2}$ ). The 16-run 2-PFDRs double the degrees of freedom of the 16-run 4-PFDRs for estimating the variance of pure error. Similar results can be found in the following 32-run 4-PFDRs.

TABLE 6: Some 16-run 4-PFDRs corresponding to the cases in Table 4 along with their measures of efficiency $D_{e}(4-\mathrm{PFDR})$ and $D_{e}(\mathrm{MD})$. Here $T$ denotes 10.

\begin{tabular}{ccccc}
\hline Case & $\boldsymbol{B}=\left\{\boldsymbol{b}_{1}, \boldsymbol{b}_{2}\right\}$ & $\boldsymbol{Z}=\left\{\boldsymbol{z}_{1}, \boldsymbol{z}_{2}\right\}$ & $D_{e}(4-\mathrm{PFDR})$ & $D_{e}(\mathrm{MD})$ \\
\hline 1 & $\{34,24\}$ & $\{1234,1234\}$ & 1.000 & 1.000 \\
2 & $\{34,24\}$ & $\{1234,123\}$ & 0.857 & 0.871 \\
3 & $\{245,1235\}$ & $\{124,124\}$ & 1.000 & 0.965 \\
4 & $\{245,1235\}$ & $\{1245,345\}$ & 0.926 & 0.938 \\
5 & $\{245,1235\}$ & $\{1245,345\}$ & 0.871 & 0.917 \\
6 & $\{245,1235\}$ & $\{12,345\}$ & 0.871 & 0.874 \\
7 & $\{245,1235\}$ & $\{12,34\}$ & 0.794 & 0.794 \\
8 & $\{245,1235\}$ & $\{1246,345\}$ & 0.926 & 0.949 \\
9 & $\{245,1235\}$ & $\{12456,345\}$ & 0.871 & 0.917 \\
10 & $\{245,1235\}$ & $\{12456,345\}$ & 0.828 & 0.876 \\
11 & $\{245,1235\}$ & $\{12456,345\}$ & 0.794 & 0.855 \\
12 & $\{245,1235\}$ & $\{26,35\}$ & 0.828 & 0.857 \\
13 & $\{245,12357\}$ & $\{1246,345\}$ & 0.871 & 0.916 \\
14 & $\{245,12357\}$ & $\{12456,345\}$ & 0.828 & 0.879 \\
15 & $\{245,12357\}$ & $\{12456,345\}$ & 0.794 & 0.855 \\
16 & $\{2457,1235\}$ & $\{1246,357\}$ & 0.794 & 0.831 \\
17 & $\{2458,12357\}$ & $\{1246,3458\}$ & 0.828 & 0.883 \\
18 & $\{2458,12357\}$ & $\{12456,3458\}$ & 0.794 & 0.855 \\
19 & $\{24589,123579\}$ & $\{1246,3458\}$ & 0.794 & 0.861 \\
20 & $\{24589,123579\}$ & $\{1246 T, 3458 T\}$ & 0.794 & 0.891 \\
\hline
\end{tabular}




\subsection{4-run 3-PFDRs and 32-run 4-PFDRs.}

We now report some 24-run and 32-run designs obtained from 3-PFDRs and 4-PFDRs, respectively. We did not calculate the D-efficiency of the corresponding Mitchell designs for 32-run cases due to computing time costs.

For Class I interactions within groups, again by Proposition 1(a), only 8 designs are available with 24 runs; these are reported in Table 8. The results for 32-run 4-PFDRs are displayed in Table 9.

For Class II interactions between groups, the specified effects are listed in Table 10 and the designs obtained from 24-run 3-PFDRs and 32-run 4-PFDRs are displayed in Tables 11 and 12, respectively.

TABLE 7: List of specified sets of within groups two-factor interactions for which there are 24-run 3-PFDRs or 32-run 4-PFDRs.

\begin{tabular}{|c|c|c|c|c|c|c|}
\hline Case & $n$ & $v$ & $p$ & $p^{\prime}$ & $\left(n_{1}, \ldots, n_{p}\right)$ & Estimable interactions \\
\hline 1 & 5 & 16 & 1 & 1 & (5) & $\{1,2,3,4,5\} \times\{1,2,3,4,5\}$ \\
\hline 2 & 6 & 13 & 2 & 1 & $(4,2)$ & $\{1,2,3,4\} \times\{1,2,3,4\}$ \\
\hline 3 & 6 & 13 & 2 & 2 & $(3,3)$ & $\{1,2,3\} \times\{1,2,3\} \cup\{4,5,6\} \times\{4,5,6\}$ \\
\hline 4 & 6 & 14 & 2 & 2 & $(4,2)$ & $\{1,2,3,4\} \times\{1,2,3,4\} \cup\{5 \cdot 6\}$ \\
\hline 5 & 6 & 17 & 2 & 1 & $(5,1)$ & $\{1,2,3,4,5\} \times\{1,2,3,4,5\}$ \\
\hline 6 & 7 & 14 & 2 & 1 & $(4,3)$ & $\{1,2,3,4\} \times\{1,2,3,4\}$ \\
\hline 7 & 7 & 15 & 3 & 2 & $(4,2,1)$ & $\{1,2,3,4\} \times\{1,2,3,4\}, \cup\{5 \cdot 6\}$ \\
\hline 8 & 7 & 17 & 2 & 2 & $(4,3)$ & $\{1,2,3,4\} \times\{1,2,3,4\} \cup\{5,6,7\} \times\{5,6,7\}$ \\
\hline 9 & 7 & 18 & 2 & 1 & $(5,2)$ & $\{1,2,3,4,5\} \times\{1,2,3,4,5\}$ \\
\hline 10 & 8 & 15 & 2 & 1 & $(4,4)$ & $\{1,2,3,4\} \times\{1,2,3,4\}$ \\
\hline 11 & 8 & 16 & 3 & 2 & $(4,2,2)$ & $\{1,2,3,4\} \times\{1,2,3,4\} \cup\{5 \cdot 6\}$ \\
\hline 12 & 8 & 17 & 3 & 3 & $(4,2,2)$ & $\{1,2,3,4\} \times\{1,2,3,4\} \cup\{5 \cdot 6\} \cup\{7 \cdot 8\}$ \\
\hline 13 & 8 & 19 & 2 & 1 & $(5,3)$ & $\{1,2,3,4,5\} \times\{1,2,3,4,5\}$ \\
\hline 14 & 9 & 13 & 2 & 1 & $(3,6)$ & $\{1,2,3\} \times\{1,2,3\}$ \\
\hline 15 & 9 & 16 & 2 & 1 & $(4,5)$ & $\{1,2,3,4\} \times\{1,2,3,4\}$ \\
\hline 16 & 9 & 16 & 3 & 2 & $(3,3,3)$ & $\{1,2,3\} \times\{1,2,3\} \cup\{4,5,6\} \times\{4,5,6\}$ \\
\hline 17 & 9 & 17 & 3 & 2 & $(2,4,4)$ & $\{1 \cdot 2\} \cup\{3,4,5,6\} \times\{3,4,5,6\}$ \\
\hline 18 & 9 & 19 & 3 & 3 & $(3,3,3)$ & $\begin{aligned}\{1,2,3\} \times & \{1,2,3\} \cup\{4,5,6\} \times\{4,5,6\} \\
& \cup\{7,8,9\} \times\{7,8,9\}\end{aligned}$ \\
\hline 19 & 9 & 20 & 2 & 1 & $(5,4)$ & $\{1,2,3,4,5\} \times\{1,2,3,4,5\}$ \\
\hline 20 & 10 & 14 & 2 & 1 & $(3,7)$ & $\{1,2,3\} \times\{1,2,3\}$ \\
\hline 21 & 10 & 15 & 3 & 2 & $(2,3,5)$ & $\{1 \cdot 2\} \cup\{3,4,5\} \times\{3,4,5\}$ \\
\hline 22 & 10 & 17 & 2 & 1 & $(4,6)$ & $\{1,2,3,4\} \times\{1,2,3,4\}$ \\
\hline 23 & 10 & 17 & 3 & 2 & $(3,3,4)$ & $\{1,2,3\} \times\{1,2,3\} \cup\{4,5,6\} \times\{4,5,6\}$ \\
\hline 24 & 10 & 18 & 3 & 2 & $(2,4,4)$ & $\{1 \cdot 2\} \cup\{3,4,5,6\} \times\{3,4,5,6\}$ \\
\hline 25 & 10 & 21 & 2 & 1 & $(5,5)$ & $\{1,2,3,4,5\} \times\{1,2,3,4,5\}$ \\
\hline
\end{tabular}


TABLE 8: Some 24-run 3-PFDRs corresponding to the possible cases in Table 7 along with their efficiency measures, $D_{e}$ (3-PFDR) and $D_{e}(\mathrm{MD})$.

\begin{tabular}{ccccc}
\hline Case & $\boldsymbol{B}=\left\{\boldsymbol{b}_{1}, \boldsymbol{b}_{2}, \boldsymbol{b}_{3}\right\}$ & $\boldsymbol{Z}=\left\{\boldsymbol{z}_{1}\right\}$ & $D_{e}(3$-PFDR $)$ & $D_{e}(\mathrm{MD})$ \\
\hline 1 & $\{14,35,25\}$ & $\{1235\}$ & 0.943 & 0.943 \\
2 & $\{146,356,256\}$ & $\{12356\}$ & 0.956 & 0.956 \\
6 & $\{146,356,256\}$ & $\{123567\}$ & 0.951 & 0.951 \\
10 & $\{3578,2568,1678\}$ & $\{124678\}$ & 0.947 & 0.947 \\
14 & $\{35789,25678,16789\}$ & $\{12345679\}$ & 0.956 & 0.956 \\
15 & $\{35789,25678,16789\}$ & $\{12345679\}$ & 0.943 & 0.943 \\
20 & $\{3578 T, 2568 T, 16789 T\}$ & $\{12345678\}$ & 0.951 & 0.951 \\
21 & $\{3578 T, 2567 T, 16789 T\}$ & $\{12345678\}$ & 0.947 & 0.947 \\
\hline
\end{tabular}

TABLE 9: Some 32-run 4-PFDRs corresponding to the cases in Table 7 along with the efficiency measure $D_{e}$.

\begin{tabular}{|c|c|c|c|}
\hline Case & $\boldsymbol{B}=\left\{\boldsymbol{b}_{1}, \boldsymbol{b}_{2}, \boldsymbol{b}_{3}\right\}$ & $\boldsymbol{Z}=\left\{\boldsymbol{z}_{1}, \boldsymbol{z}_{2}\right\}$ & $D_{e}(4-\mathrm{PFDR})$ \\
\hline 1 & $\{14,35,25\}$ & $\{1235,1235\}$ & 1.000 \\
\hline 2 & $\{146,356,256\}$ & $\{12356,12356\}$ & 1.000 \\
\hline 3 & $\{46,35,256\}$ & $\{1246,1346\}$ & 0.948 \\
\hline 4 & $\{146,356,256\}$ & $\{12356,23456\}$ & 0.952 \\
\hline 5 & $\{146,356,256\}$ & $\{12356,456\}$ & 0.960 \\
\hline 6 & $\{146,356,256\}$ & $\{123567,123567\}$ & 1.000 \\
\hline 7 & $\{146,356,2567\}$ & $\{12356,1367\}$ & 0.955 \\
\hline 8 & $\{1467,3567,256\}$ & $\{123567,234567\}$ & 0.885 \\
\hline 9 & $\{146,356,256\}$ & $\{123567,456\}$ & 0.926 \\
\hline 10 & $\{3578,2568,1678\}$ & $\{124678,34678\}$ & 1.000 \\
\hline 11 & $\{3578,25678,1678\}$ & $\{1234567,4568\}$ & 1.000 \\
\hline 12 & $\{3578,25678,1678\}$ & $\{1234567,4568\}$ & 0.960 \\
\hline 13 & $\{3578,2568,1678\}$ & $\{1245678,34678\}$ & 0.896 \\
\hline 14 & $\{35789,25678,16789\}$ & $\{12345679,12345679\}$ & 1.000 \\
\hline 15 & $\{35789,25678,16789\}$ & $\{12345679,12345679\}$ & 1.000 \\
\hline 16 & $\{46789,359,2568\}$ & $\{1235679,1578\}$ & 0.958 \\
\hline 17 & $\{3578,2567,16789\}$ & $\{12345678,4579\}$ & 0.960 \\
\hline 18 & $\{4678,357,25678\}$ & $\{1345789,2346789\}$ & 0.833 \\
\hline 19 & $\{35789,25678,16789\}$ & $\{123456789,1234689\}$ & 0.871 \\
\hline 20 & $\{3578 T, 2568 T, 16789 T\}$ & $\{12345678,12345678\}$ & 1.000 \\
\hline 21 & $\{3578 T, 2567 T, 16789 T\}$ & $\{12345678,12345678\}$ & 1.000 \\
\hline 22 & $\{3578 T, 2568 T, 16789 T\}$ & $\{123456789,23459 T\}$ & 0.960 \\
\hline 23 & $\{46789 T, 359 T, 2568 T\}$ & $\{1234569 T, 1578\}$ & 0.960 \\
\hline 24 & $\{3578,2567,16789\}$ & $\{12345678,4579 T\}$ & 0.926 \\
\hline 25 & $\{3578 T, 2568 T, 16789 T\}$ & $\{123456789,2349 T\}$ & 0.848 \\
\hline
\end{tabular}


TABLE 10: List of specified sets of between groups two-factor interactions for which there are 24-run 3-PFDRs or 32-run 4-PFDRs.

\begin{tabular}{|c|c|c|c|c|c|c|}
\hline Case & $n$ & $v$ & $p$ & $p^{\prime}$ & $\left(n_{1}, \ldots, n_{p}\right)$ & Estimable interactions \\
\hline 1 & 6 & 15 & 2 & 2 & $(4,2)$ & $\{1,2,3,4\} \times\{5,6\}$ \\
\hline 2 & 6 & 16 & 2 & 2 & $(3,3)$ & $\{1,2,3\} \times\{4,5,6\}$ \\
\hline 3 & 7 & 14 & 2 & 2 & $(1,6)$ & $\{1\} \times\{2,3,4,5,6,7\}$ \\
\hline 4 & 7 & 14 & 3 & 2 & $(3,2,2)$ & $\{1,2,3\} \times\{4,5\}$ \\
\hline 5 & 7 & 17 & 3 & 2 & $(3,3,1)$ & $\{1,2,3\} \times\{4,5,6\}$ \\
\hline 6 & 7 & 18 & 2 & 2 & $(2,5)$ & $\{1,2\} \times\{3,4,5,6,7\}$ \\
\hline 7 & 8 & 15 & 3 & 2 & $(3,2,2)$ & $\{1,2,3\} \times\{4,5\}$ \\
\hline 8 & 8 & 16 & 2 & 2 & $(1,7)$ & $\{1\} \times\{2,3,4,5,6,7,8\}$ \\
\hline 9 & 8 & 21 & 2 & 2 & $(2,6)$ & $\{1,2\} \times\{3,4,5,6,7,8\}$ \\
\hline 10 & 8 & 21 & 3 & 2 & $(3,4,1)$ & $\{1,2,3\} \times\{4,5,6,7\}$ \\
\hline 11 & 8 & 24 & 2 & 2 & $(3,5)$ & $\{1,2,3\} \times\{4,5,6,7,8\}$ \\
\hline 12 & 9 & 14 & 3 & 2 & $(2,2,5)$ & $\{1,2\} \times\{3,4\}$ \\
\hline 13 & 9 & 16 & 3 & 2 & $(2,3,4)$ & $\{1,2\} \times\{3,4,5\}$ \\
\hline 14 & 9 & 18 & 2 & 2 & $(1,8)$ & $\{1\} \times\{2,3,4,5,6,7,8,9\}$ \\
\hline 15 & 9 & 18 & 3 & 2 & $(2,4,3)$ & $\{1,2\} \times\{3,4,5,6\}$ \\
\hline 16 & 9 & 19 & 3 & 2 & $(3,3,3)$ & $\{1,2,3\} \times\{4,5,6\}$ \\
\hline 17 & 9 & 20 & 3 & 2 & $(2,5,2)$ & $\{1,2\} \times\{3,4,5,6,7\}$ \\
\hline 18 & 9 & 22 & 3 & 2 & $(4,3,2)$ & $\{1,2,3,4\} \times\{5,6,7\}$ \\
\hline 19 & 9 & 24 & 2 & 2 & $(2,7)$ & $\{1,2\} \times\{3,4,5,6,7,8,9\}$ \\
\hline 20 & 10 & 15 & 3 & 2 & $(2,2,6)$ & $\{1,2\} \times\{3,4\}$ \\
\hline 21 & 10 & 17 & 3 & 2 & $(2,3,5)$ & $\{1,2\} \times\{3,4,5\}$ \\
\hline 22 & 10 & 20 & 2 & 2 & $(1,9)$ & $\{1\} \times\{2,3,4,5,6,7,8,9, T\}$ \\
\hline 23 & 10 & 20 & 3 & 2 & $(3,3,4)$ & $\{1,2,3\} \times\{4,5,6\}$ \\
\hline 24 & 10 & 21 & 3 & 2 & $(2,5,3)$ & $\{1,2\} \times\{3,4,5,6,7\}$ \\
\hline 25 & 10 & 23 & 3 & 2 & $(4,3,3)$ & $\{1,2,3,4\} \times\{5,6,7\}$ \\
\hline 26 & 10 & 23 & 3 & 2 & $(6,2,2)$ & $\{1,2,3,4,5,6\} \times\{7,8\}$ \\
\hline
\end{tabular}

TABLE 11: Some 24-run 3-PFDRs corresponding to the possible cases of Table 10 along with their efficiency measures $D_{e}(3-\mathrm{PFDR})$ and $D_{e}(\mathrm{MD})$.

\begin{tabular}{ccccc}
\hline Case & $\boldsymbol{B}=\left\{\boldsymbol{b}_{1}, \boldsymbol{b}_{2}, \boldsymbol{b}_{3}\right\}$ & $\boldsymbol{Z}=\left\{\boldsymbol{z}_{1}\right\}$ & $D_{e}(3$-PFDR $)$ & $D_{e}(\mathrm{MD})$ \\
\hline 2 & $\{146,356,256\}$ & $\{12356\}$ & 0.943 & 0.943 \\
3 & $\{1467,356,2567\}$ & $\{3467\}$ & 0.951 & 0.951 \\
4 & $\{1467,356,2567\}$ & $\{12356\}$ & 0.951 & 0.951 \\
7 & $\{14678,3568,2567\}$ & $\{12356\}$ & 0.947 & 0.947 \\
8 & $\{14678,3568,2567\}$ & $\{1235678\}$ & 0.943 & 0.943 \\
12 & $\{16789,35689,25789\}$ & $\{12345678\}$ & 0.951 & 0.951 \\
13 & $\{14678,3568,25679\}$ & $\{123568\}$ & 0.943 & 0.943 \\
20 & $\{16789,356 T, 25789\}$ & $\{12345679\}$ & 0.947 & 0.947 \\
\hline
\end{tabular}


TABLE 12: Some 32-run 4-PFDRs corresponding to the cases of Table 10 along with their efficiency measure $D_{e}$. Here $T$ denotes 10 .

\begin{tabular}{|c|c|c|c|}
\hline Case & $\boldsymbol{B}=\left\{\boldsymbol{b}_{1}, \boldsymbol{b}_{2}, \boldsymbol{b}_{3}\right\}$ & $\boldsymbol{Z}=\left\{\boldsymbol{z}_{1}, \boldsymbol{z}_{2}\right\}$ & $D_{e}$ (4-PFDR) \\
\hline 1 & $\{35,245,145\}$ & $\{56,456\}$ & 0.912 \\
\hline 2 & $\{146,356,256\}$ & $\{12356,12356\}$ & 1.000 \\
\hline 3 & $\{1467,356,2567\}$ & $\{3467,3467\}$ & 1.000 \\
\hline 4 & $\{1467,356,2567\}$ & $\{12356,12356\}$ & 1.000 \\
\hline 5 & $\{146,356,256\}$ & $\{123567,346\}$ & 0.960 \\
\hline 6 & $\{356,24,145\}$ & $\{12347,2347\}$ & 0.857 \\
\hline 7 & $\{14678,3568,2567\}$ & $\{12356,12356\}$ & 1.000 \\
\hline 8 & $\{14678,3568,2567\}$ & $\{1235678,1235678\}$ & 1.000 \\
\hline 9 & $\{4678,1578,2568\}$ & $\{123578,23458\}$ & 0.820 \\
\hline 10 & $\{1467,356,2567\}$ & $\{12478,45\}$ & 0.848 \\
\hline 11 & $\{1467,3568,2567\}$ & $\{12358,2345\}$ & 0.794 \\
\hline 12 & $\{16789,35689,25789\}$ & $\{12345678,12345678\}$ & 1.000 \\
\hline 13 & $\{14678,3568,25679\}$ & $\{123568,123568\}$ & 1.000 \\
\hline 14 & $\{14678,3568,2567\}$ & $\{12356789,1235678\}$ & 0.926 \\
\hline 15 & $\{14789,3589,2579\}$ & $\{1235689,5678\}$ & 0.926 \\
\hline 16 & $\{14678,3568,25679\}$ & $\{12356789,459\}$ & 0.896 \\
\hline 17 & $\{15679,35689,2579\}$ & $\{1234679,146789\}$ & 0.841 \\
\hline 18 & $\{1678,3568,257\}$ & $\{145689,467\}$ & 0.828 \\
\hline 19 & $\{14678,3568,25679\}$ & $\{12356789,4569\}$ & 0.794 \\
\hline 20 & $\{16789,3569 T, 25789\}$ & $\{12345679,12345679\}$ & 1.000 \\
\hline 21 & $\{16789 T, 3569 T, 25789\}$ & $\{1247,145679\}$ & 0.960 \\
\hline 22 & $\{14678,3568,2567 T\}$ & $\{123456789,1356789 T\}$ & 0.871 \\
\hline 23 & $\{14678,356,2567 T\}$ & $\{124679,15689\}$ & 0.871 \\
\hline 24 & $\{16789 T, 3569 T, 25789\}$ & $\{1247,145679\}$ & 0.848 \\
\hline 25 & $\{1678 T, 356 T, 2578\}$ & $\{1249,14568\}$ & 0.810 \\
\hline 26 & $\{12679,2356,2578\}$ & $\{1234567,45679 T\}$ & 0.810 \\
\hline
\end{tabular}

\section{CONCLUDING REMARKS}

We have proposed a systematic method for constructing two-level factorial designs of userspecified resolution with partial duplication from parallel flats designs. The 3-PFDRs and 4PFDRs we obtained not only have satisfactory efficiency in estimating the specified factorial effects but also degrees of freedom for estimating the variance of pure error. The covariance structure of these designs is known and very simple. This appealing property ensures that statistical inference and interpretation will be easier. Therefore, we believe that these designs should prove useful in practice.

There are several publications on identifying location effects and dispersion effects by using unreplicated two-level factorial designs. See Box \& Meyer (1986), Bergman \& Hynên (1997), Pan (1999), Liao \& Iyer (2000) and McGrath \& Lin (2001). As discussed in Pan (1999) and McGrath \& Lin (2001), the nature of unreplicated designs inevitably leads to the problem that dispersion effects are confounded with location effects. As a result, dispersion effects cannot be identified efficiently. Pan (1999) thus strongly suggests that dispersion effects should be estimated from the pure replicates and illustrates that dispersion effects can be identified using an 
economical twice-replicated fractional factorial experiment. So $f$-PFDRs can be good, practical choices for studying location effects and dispersion effects simultaneously. This will be our next research project.

\section{ACKNOWLEDGEMENTS}

We thank the Editor, an Associate Editor and two referees for helpful comments that significantly improved the content and presentation of this article.

\section{REFERENCES}

B. Bergman \& A. Hynên (1997). Dispersion effects from fractional factorial designs in the $2^{k-p}$ series. Technometrics, 39, 191-198.

G. E. P. Box \& R. D. Meyer (1986). Dispersion effects from fractional designs. Technometrics, 28, 19-27.

F. S. Chai \& C. T. Liao (2001). Three parallel flats designs for two-level experiments. Australian \& New Zealand Journal of Statistics, 43, 113-119.

O. Dykstra Jr. (1959). Partial duplication of factorial experiments. Technometrics, 1, 63-75.

M. F. Franklin \& R. A. Bailey (1977). Selecting defining contrasts and confounded effects in two-level experiments. Applied Statistics, 27, 321-326.

Z. Galil \& J. Kiefer (1980). Time and space-saving computer methods, related to Mitchell's DETMAX, for finding D-optimal designs. Technometrics, 22, 301-313.

C. T. Liao \& H. K. Iyer (2000). Optimal $2^{n-p}$ fractional factorial designs for dispersion effects under a location-dispersion model. Communications in Statistics: Theory and Methods, 29, 823-835.

C. T. Liao, H. K. Iyer \& D. F. Vecchia (1996). Orthogonal designs of two-level user-specified resolution where $N \neq 2^{k}$. Technometrics, 38, 342-353.

R. N. McGrath \& D. K. J. Lin (2001). Confounding of location and dispersion effects in unreplicated fractional factorials. Journal of Quality Technology, 33, 129-139.

T. J. Mitchell (1974). An algorithm of D-optimal experimental designs. Technometrics, 16, 203-210.

G. Pan (1999). The impact of unidentified location effects on dispersion effects identification from unreplicated factorial designs. Technometrics, 41, 313-326.

J. G. Pigeon \& P. R. McAllister (1989). A note on partially replicated orthogonal main-effect plans. Technometrics, 31, 249-251.

SAS Institute (2002). SAS/QC User's Guide: Release 8.1 Edition. SAS Institute, Cary, North Carolina.

J. N. Srivastava, D. A. Anderson \& J. Mardekian (1984). Theory of factorial designs of parallel flats type. I. The coefficient matrix. Journal of Statistical Planning and Inference, 9, 229-252.

J. N. Srivastava \& F. Li (1996). Orthogonal designs of parallel flats type. Journal of Statistical Planning and Inference, 53, 261-283.

W. J. Welch (1984). Computer-aided design of experiments for response estimation. Technometrics, 26, 217-224.

C. F. J. Wu \& M. Hamada (2000). Experiments: Planning, Analysis, and Parameter Design Optimization. Wiley, New York.

Received 18 November 2002

Accepted 30 April 2004
Chen-Tuo LIAO: ctliao@ntu.edu.tw

Division of Biometry Institute of Agronomy National Taiwan University, Taipei, Taiwan 10673

Feng-Shun CHAI: fschai@ stat.sinica.edu.tw Institute of Statistical Science Academia Sinica

Taipei, Taiwan 11529 